Biol. Neonat. 1959;1:1-7

\title{
Foreword - Avant-propos - Vorwort
}

The most memorable characteristic of the complex sequence of events resulting in the growth, development and birth of the human fetus is the fact that, as a rule, the process unfolds normally and without mishap.

The normal mechanisms of fetal and neonatal life should therefore constitute the leading subject of scientific research. In spite of the many and excellent papers published, pertaining to this subject, problems still abound in this field. Safe methods of exploring the human fetus in utero have not yet been devised. We are indebted to Barer oft for his major contribution to our understanding of the respiratory physiology of the animal fetus. Most of the available knowledge on physiology of the newborn and the fetus are summarized in C.A.Smíth-'s and Wíndle-'s books. Many documents are now available concerning the extra-uterine life of the human fetus or the non-viable premature of three to six months gestational age. But we are only getting a fragmentary picture of the clinical and biochemical aspects of the different stages of fetal life. Only a greater familiarity with the problems of the unborn human will enable us to avoid the acquired congenital defects and permanent lesions of the brain, which cease to be amenable to treatment after birth and turn the joys of parenthood into one of the most heartrending experiences a family can face. Let us remember the hundreds of thousands of brain-damaged children born yearly throughout the world, who are rejected by their environment and whose livelihood is assured only in a few highly developed countries.

Clinical observation is still the mainstay of studies of this phase of life. Students of the newborn know that even with years of experience to fall back on, the meaning of symptoms is difficult to interpret: a picture of respiratory distress may be due to three or four

Biologia Nconatormii, Vol. 1, No. 1 (1959) 1

2

Foreword

different causes, all of which require different treatment. Clinical findings are often related to difficulty with a given biological or physiological process.

The name of Andre Thomas must be quoted in this context. He has extended the domain of neurological examination enabling it to encompass the neonatal age and has shown its fundamental significance. Clinical observation so far overcomes the accomplishments of the pathological, neurophysiological and neurochemical approach that new hypotheses concerning growth and development are being suggested at every turn of the road. Progress and maturation is far from being the only course followed by this particular form of life. The case of the greater resistance of the fetus and premature infant to anoxia compared to the term newborn is an example of a regressive type of evolution.

The main problem facing the investigator of neonatal pediatrics is the definition of normalcy. This implies more than the collection of figures, chemical data and standard deviations and it is a far too common practice among authors to consider their task completed once these data are made available. Effective research also implies the constant confrontation of clinical facts and biological data and an awareness of the importance of findings falling beyond the limits of the 
standard deviation. Extreme marginal findings far from being neglected should focus all our attention, and as an example illustrating this problem we wish to call to mind the case of extreme figures of oxygen saturation values in the arterial cord blood, which contribute information of the highest interest in the fetus as well as in the normal newborn. The gradual increase of our knowledge of the main characteristics of normalcy will enable us to map accurately the limits of the pathological. More than any other living being, the newborn compels us to define the characteristics of the normal individual.

A heavy task awaits the investigator, fully aware of the fact that a major part of our destiny is decided within the first nine months of our life.

A closer cooperation and coordination of efforts between the widely diverging branches of medical and non-medical science is highly desirable. A multiplication of exchanges of information between countries which either do not communicate on a large scale or even completely ignore each other, is essential. The excuses of existing linguistic barriers or of narrow compartimentation into limited spe-

Foreword

3

cialities should cease to be acceptable justifications for ignoring the work of other investigators at a time when fetal mortality and pathology is one of the major unsolved problems of medical science.

Two types of papers will be accepted in this journal: on the one hand, papers presenting a survey of ascertained or nearly certain facts in the form of general review articles; on the other hand, papers describing original research, and for these the policy will be that a good idea and satisfactory preliminary report will be acceptable even if no formal conclusion is reached, research consisting often in raising problems before solving them.

The International Children's Centre has enabled this journal to be born. This new edition, we hope, will enable it to live and thrive.

A.Mínkowskí, M.D.

Avant-propos

II est remarquable que, dans tout ce qui concourt à Гédification de $\Gamma$ homme et, plus encore, à son arrivée dans le monde aérien, les évènements se passent en regie bien et sans incidents.

Cette constatation impose une premiere recherche; celle des mé-canismes normaux de la vie embryonnaire, fcetale et néonatale. Or dans ce domaine, malgré l'abondance et la qualité des travaux, tout est encore à faire. On n'a, en effet, pas encore trouvé le moyen d'explorer sans danger le foetus humain en place dans $\Gamma$ utérus maternel. Nous avons beaucoup appris, grace à Barcroft, ce qu'est la physiologie respiratoire du foetus animal; les livres de C. A. Smith et de Windle ont, les premiers, rassemblé les notions de physiologie néo-na-tale et fcetale; nous avons aussi maintenant de nombreux documents sur le foetus humain (vivant ou premature inviable) de 3 à 6 mois hors de Гutérus maternel, mais nous ne faisons encore qu'entrebaîller la porte du domaine de Гexploration, biologique et clinique, de la vie fcetale, telle qu'elle est, à toutes ses étapes. C'est pourtant la seule connais-sance de Гêtre humain prenatal qui permettra de prévenir les malformations congénitales acquises et surtout la survenue des lesions 4

Avant-prcpos

cérébrales definitives. Celles-ci ne sont déjà plus accessibles au trai-tement lorsque Tenfant naît et feront de Гenfance au lieu d'une joie, une des plus douloureuses épreuves qui puisse être imposée à une mere, à une famille. On oublie peut-être que, chaque année, naissent ainsi dans le 
monde plusieurs centaines de milliers d'individus que la société rejette, et que seuls, quelques pays, à Гesprit élevé, prennent à leur charge.

La clinique est, à cette période de la vie, encore à faire; tous ceux qui passent des années à observer les nouveau-nés savent comme il est difficile de connaître le sens des symptômes, comment un même tableau de souffrance respiratoire peut ressortir à 3 ou 4 causes différentes justiciables de traitements très divers, comment il est souvent impossible de relier ce que $\Gamma$ on voit à des données precises, biologiques ou physiopathologiques.

On ne peut, dans ce domaine, passer sous silence le nom d-Andre Thomas: il a rendu à la séméiologie neurologique tout son sens, à cette époque du développement; la clinique est là, tenement en avance sur les données de Гanatome-pathologie, de la neuro-physio-logie et de la neuro-chimie, qu'elle incite à chercher, qu'elle suggère à chaque pas, certaines hypotheses sur les phénomènes du développement. Tout n'est d'ailleurs pas que progrès et maturation à sens unique dans ce domaine, si particulier de l'existence; la resistance du premature et du fæus à Гanoxie, plus grande que celle du nouveau-né à terme, en est un exemple.

Nous avions dit plus haut qu'il fallait avant tout - dans ce domaine - préciser le normal. II est nécessaire pour cela - non pas de rassembler quelques chiffres - quelques données biologiques et d'en calculer la deviation standard, ce dont de trop nombreux chercheurs semblent se contenter, mais encore:

$1^{\circ}$ de confronter constamment les faits cliniques avec les données biologiques;

$2^{\circ}$ de considérer avec autant d'importance que la deviation standard - la marge des extremes.

Ces valeurs extremes au lieu d'etre rejetées, doivent au contraire retenir toute notre attention; car on peut les observer chez des foetus ou des nouveau-nés normaux (par exemple les chiffres de saturation oxygénée du sang artériel fætal dans le cordon). Plus nous étendons ainsi notre connaissance sur l'essentiel du normal, plus nous saurons comme il peut se confondre avec le pathologique; le nouveau-né nous oblige, plus que tout autre, à préciser les caractères de l'individu normal.

Avant-propos

5

La tâche reste done entière à ceux qui cherchent, et qui savent qu'une grande partie de notre destin se joue dans ces neuf premiers mois.

Une cooperation chaque jour plus grande s'impose pour réunir dans cet effort des disciplines si différentes, voire non médicales, de relier des pays qui communiquent trop peu entre eux ou qui s'ignorent même; les prétextes de I'incompréhension des langues d'origine, du cloisonnement des spécialités ne doivent plus être invoqués par les chercheurs à Гignorance des travaux d'autrui, à une époque où la mortalité et la pathologie fætale et néonatale restent un des grands problèmes non résolus de la médecine.

Nous voudrions ouvrir ici nos colonnes à deux sortes d'articles:

- des travaux scientifiques qui représenteraient une somme deconnaissances à peu près vérifiées, sous forme de revue générale;

- des travaux de recherche originale; pour ceux-ci nous pensonsque, si l'idée est bonne et le debut du travail satisfaisant, il n'est pasnécessaire qu'il y ait une conclusion; le recherche consiste souventà poser un problème sans chercher à la résoudre.

Le Centre International de $\Gamma$ Enfance avait permis à ce Journal de naître; puisse la nouvelle edition lui permettre de vivre et de rayonner.

Docteur A.Mínkowski

Vorwort 
Es ist bemerkenswert, daß Entwicklung, Wachstum und schließ-lich Geburt der menschlichen Frucht in der Regel normal und ohne Zwíschenfälle verlaufen.

Die Erkennung der normalen Lebensfunktionen des Embryos, des Foetus und des Neugeborenen muß zunächst das Streben unserer wis-senschaftlichen Forschung sein. Trotz der großen Zahl ausgezeichne-ter Arbeiten, die über dieses Gebiet schon vorliegen, ist eine Fülle von Fragen noch offen. Gibt es doch bisher weder eine Methode noch eine Möglichkeit, ohne Gefahren den menschlichen Fætus im mutter-lichen Uterus selbst zu untersuchen und zu erforschen. Dank der Arbeiten von Barcroft wissen wir zwar viel über die Atmungsphysiologie

6

Vorwort

des tierischen Foetus, auch liegen bereits zahlreiche Forschungsergeb-nisse über das extrauterine Verhalten von 3 bis 6 Monate alten menschlichen Foeten vor, aber biologisch wie klinisch ist seine Ent-wicklung bisher ein Geheimnis geblieben.

Nur sicheres Wissen über das pränatale menschliche Leben wird es uns möglich machen, erworbene angeborene Mißbildungen, und vor allem bleibende Gehirnläsionen, zu verhüten, die nach der Geburt nicht mehr behandelt werden können und die die Geburt eines Kindes statt zu einer Queue der Freude für Mutter und Familie zu einer dauernden schmerzlichen Prüfung werden lassen.

Wir müssen an die Hunderttausende von geistesschwachen Kindern denken, die jährlich das Licht der Welt erblicken und denen, von der übrigen Gesellschaft verstoßen, nur in wenigen, sozial hochentwickel-ten Staaten Schutz und Fürsorge zuteil wird.

Die klinische Erforschung des pränatalen Lebensabschnitts $b^{1 / 8 g t}$ also noch vor uns. Wer sich schon jahrelang mit seinem Studium be-schäftigt hat, weiß, wie schwer es ist, die Bedeutung der Symptome richtig zu erfassen. Gleichartige Atemstörungen zum Beispiel können drei bis vier verschiedene Ursachen haben und sehr unterschiedüche Behandlung erfordern; klinische Befunde sind oft schwer mit den vorliegenden biologischen oder physio-pathologischen Vorgängen in Ubereinstimmung zu bringen.

In diesem Zusammenhang verdient vor allem Andre Thomas be-sondere Erwähnung. Er hat die Möglichkeit neurologischer Prüfung und Symptomatologie in dieser Entwicklungsphase und ihre funda-mentale Bedeutung gezeigt. Damit wurde nicht nur ein Fortschritt über die neurophysiologischen, neuro-chemischen und anatomisch-pathologischen Methoden hinaus erzielt, sondern in vielen Fallen er-gaben sich auch Anregungen zu Hypothesen über gewisse Phänomene der Entwicklung.

Fortschritt und Reifung sind aber nicht allein in dieser Phase der Entwicklung beachtenswert.In der Widerstandskraft der Foeten und Frühgeburten gegenüber einer Anoxie zeigt sich beispielsweise eine Überlegenheit im Vergleich zu den am normalen Termin Ge-borenen. Wie wir festgestellt haben, muß sich die Forschung um die genaue Kenntnis des Normalen bemühen. Man darf sich jedoch nicht, wie zu viele Forscher, damit begnügen, Zahlen, Aufstellungen und biolo-gische Daten zusammenzustellen und Standardabweichungen zu berechnen. Wichtig ist vielmehr:

Vorwort

7

Die klinischen Befunde den biologischen G-egebenheiten gegen-überzustellen.

Die extremen Befunde ebenso sorgfältig wie die Standardabwei-chungen zu beachten.

Extreme Befunde können wir sowohl bei den Fæten wie bei den normalen Neugeborenen beobachten, wie zum Beispiel die Werte für die Sauerstoffsättigung im arteriellen Blut der 
Nabelschnur. Die zu-nehmende Kenntnis der Hauptcharakteristiken des Normalen wird es uns erleichtern, die Übergänge zum Pathologischen zu finden.

Die Aufgabe stellt sich in ihrer ganzen Tragweite den Forschern, die sich bewußt sind, daß unser Schicksal zum großen Teil in den ersten 9 Monaten unseres Lebens bestimmt wird. Sie soUten in immer engerer Zusammenarbeit auf den verschiedenen medizinischen und nicht-medizinischen Fachgebieten ihre Erfahrungen international aus-tauschen, so daß überall die

Forschungsergebnisse auch aus anderen Ländern bekannt werden. Sprachliche Schwierigkeiten müssen ebenso wie dieFolgen zuspezialisierterEinschränkungenüberwunden werden, damit die fætale bzw. neonatale Pathologie und Sterblichkeit auf-hört, eines der großen ungelösten Probleme der Medizin zu sein.

Unsere Zeitschrift will vor allem zwei Gruppen von Arbeiten ver-öífentlichen:

Ubersichtsreferate von sicheren oder annähernd sicheren Erkennt-nissen.

Originalarbeiten aus den verschiedenen Forschungsgebieten. Diese müssen nicht unbedingt eindeutige Resultate aufweisen. In der For-schung ist schon die Problemstellung wertvoll, ohne daß zugleich eine Lösung gelingt.

D. Das Centre International de $\Gamma$ Enfance hat die Gründung dieser Zeitschrift ermöglícht. Möge ihr die Neugestaltung zu weiterem Er-folg und Eingang in alle interessierten Kreise verhelfen. Dr. A.Mínkowskí 